Bala Amala Kannan, Jacquelyn Dawn Parente*, Sabine Hensler, Claudia Kuhlbach, Margareta M. Mueller, and Knut Möller

\title{
Parameter sensitivity analysis on a mathematical model of reepithelialization
}

https://doi.org/10.1515/cdbme-2019-0021

Abstract: Reepithelialization is the single requirement to define a wound as healed when the barrier function of the skin is restored. An existing reepithelialization mathematical model (RM) simulates wound healing in vitro. This work performs a parameter sensitivity analysis on an existing RM to see how robust the model is for changing wound healing rates for application to chronic wounds (inhibition) and wound healing therapies (activation). The existing RM balances the optimal distance between cells and basal membrane segments (BMs) according to the calculation of intercellular pressure and adhesion force. The RM mimics cell behavior and their interaction by passive migration, which is the displacement of cells from its initial position. First, this work reproduces the RM. The initial case recreates the interaction of a cell with its surrounding cells, while the second case recreates the interaction of the cell with its nearest BMs. These two cases were implemented in MATLAB to estimate optimal distance, intercellular pressure, an adhesive force between cells and the BMs. The analysis computes movement vectors and new positions of each cell at different time steps. Parameter sensitivity analysis was then conducted on the adhesion coefficient, where the original value in the RM was unknown. The results obtained at the assumed original parameter values are similar to the existing RM. As a result of the parameter sensitivity analysis, increasing the adhesion coefficient increases cell movement. High basal adhesion causes passive movement of cells, which in the simulation results is seen as a cellular movement towards wound closure. The existing RM is robust to changing adhesion coefficient values which change the rate of the advancing reepithelialization front. Future work includes fitting adhesion coefficient parameter values to an in vitro wounded tissue visualized by live dyes in treatment therapy experiments.

\footnotetext{
Bala Amala Kannan, Institute of Technical Medicine, Furtwangen University, Jakob-Kienzle-Straße 17, 78054

Villingen-Schwenningen, Germany

*Corresponding author: Jacquelyn Dawn Parente, Knut Möller, Institute of Technical Medicine, Furtwangen University, e-mail: pjd@hs-furtwangen.de and moe@hs-furtwangen.de Sabine Hensler, Claudia Kuhlbach, Margareta M. Mueller, Molecular Cell Biology Laboratory and Institute of Technical Medicine, Furtwangen University
}

Keywords: cell migration, parameter sensitivity analysis, reepithelialization model, wound healing, wound modelling.

\section{Introduction}

In the human body, the skin acts as a barrier with essential functions. When these functions are intruded, wound healing occurs either naturally or through receiving aid from some activating factors. Reepithelialization is an important stage in wound healing, which includes migration, proliferation, and differentiation. Restoration of the intact epidermal barrier occurs in this stage [1]. Thereby, forming a shield in the wound bed. The given computational model performs a crucial function of creating such barrier [2].

Parameter sensitivity analysis is a technique used to analyze the significance of the dependent parameters, due to variations made in the independent parameter. In this paper, the model performs cell migration which includes two movement types: cell-basal membrane interaction and cell-cell interaction. The passive migration of the cells involves intercellular pressure, cohesion force, and adhesion to the basal membrane. The cohesion and adhesion coefficients are the independent parameters which modulate the strength of cohesion and adhesion force. Varying these parameters show a great impact on cell movement. Therefore, sensitivity analysis is conducted on these parameters and the outcome is observed. This work suggests coefficients which provide a better result for cell migration along with its optimal balancing to build intact tissue for wound closure.

\section{Methods}

\subsection{Reepithelialization model}

An existing reepithelialization model discloses a mechanism, which brings out the structure of reepithelialization [2]. This model re-establishes a robust epidermal layer to the wound closure. Cell migration is achieved by the lifting mechanism of edge cells, thus a multi-layered epidermis was constructed to create a shield. The migration of basal cells along with its neighboring cells towards the closure is shown in the simula- 
tion result, and thereby create a robust shield on the wound bed. This result resembles the hypothesis of a sliding mechanism. To justify this a computational modeling was performed.

Here, a cell center-based biomechanical model was reproduced that optimizes the distance between adjacent cells and cell migration to the wound closure [2]. Passive cell migration occurs when the optimization is performed for all the cells involved in the simulation. The calculation of intercellular pressure, cell-cell cohesion, and adhesion to the basal membrane aids in balancing the distance.

To balance the optimal distance between two cells, the calculation follows [2]:

$\mathrm{d}_{\mathrm{opt}}\left(\mathbf{r}_{\mathrm{c}}, \mathbf{r}_{\mathrm{n}}\right)=\left\|\frac{\hat{\mathbf{v}}_{\mathrm{cn}}}{\sqrt{\left[\frac{\hat{\mathrm{v}}_{\mathrm{cn} 1}^{2}}{a_{\mathrm{c}}^{2}}+\frac{\hat{\mathrm{v}}_{\mathrm{cn} 2}^{2}}{a_{\mathrm{c}}^{2}}\right]}}\right\|_{2}+\left\|\frac{\hat{\mathbf{v}}_{\mathrm{nc}}}{\| \sqrt{\left[\frac{\hat{\mathrm{v}}_{\mathrm{nc} 1}^{2}}{a_{\mathrm{n}}^{2}}+\frac{\hat{\mathrm{v}}_{\mathrm{nc} 2}^{2}}{a_{\mathrm{n}}^{2}}\right]}}\right\|_{2}$

$$
\begin{aligned}
& \hat{\mathbf{v}}_{\mathrm{cn}}=\frac{1}{\| \mathbf{r}_{\mathrm{n}}-\mathbf{r}_{\mathrm{c} \|_{2}}} \times\left(\mathbf{r}_{\mathrm{n}}-\mathbf{r}_{\mathrm{c}}\right) \\
& \hat{\mathbf{v}}_{\mathrm{nc}}=\frac{1}{\left\|\mathbf{r}_{\mathrm{c}}-\mathbf{r}_{\mathrm{n}}\right\|_{2}} \times\left(\mathbf{r}_{\mathrm{c}}-\mathbf{r}_{\mathrm{n}}\right)
\end{aligned}
$$

where the $r_{c}$ and $r_{n}$ are the cell's center of masses. The radius of the cells are $a_{c}$ and $a_{n}$. Intercellular pressure and cell-cell cohesion are calculated between overlapping cells and adjacent cells as follows:

$$
\mathrm{P}\left(\mathbf{r}_{\mathrm{c}}, \mathbf{r}_{\mathrm{n}}\right)= \begin{cases}\frac{\delta_{\mathrm{p}} \times \mathrm{d}_{\mathrm{opt}}\left(\mathbf{r}_{\mathrm{c}}, \mathbf{r}_{\mathrm{n}}\right)}{\left\|\mathbf{v}_{n c}\right\|_{2}} & \text { if } \mathbf{r}_{\mathrm{c}} \neq \mathbf{r}_{\mathrm{n}} \\ 0 & \text { otherwise }\end{cases}
$$

$\mathrm{F}\left(\mathbf{r}_{\mathrm{c}}, \mathbf{r}_{\mathrm{n}}\right)= \begin{cases}\frac{\mathrm{d}\left(\mathbf{r}_{\mathrm{c}}, \mathbf{r}_{\mathrm{n}}\right)}{\left\|\mathbf{v}_{n c}\right\|_{2}} & \text { if } \mathbf{r}_{\mathrm{c}} \neq \mathbf{r}_{\mathrm{n}} \wedge\left\|\mathbf{v}_{\mathrm{nc}}\right\|_{2}<\delta_{\mathrm{ad}} \times \mathrm{d}\left(\mathbf{r}_{\mathrm{c}}, \mathbf{r}_{\mathrm{n}}\right) \\ 0 & \text { otherwise, }\end{cases}$

The results from the above equations are used in the calculation of movement vector:

$$
\mathrm{m}\left(\mathbf{r}_{\mathrm{c}}, \mathbf{r}_{\mathrm{n}}\right)= \begin{cases}(\mathrm{P}-1)) \times \mathbf{v}_{\mathrm{nc}} & \text { if } \mathrm{P}>1 \\ -\mathrm{k}(1-\mathrm{F}) \times \mathbf{v}_{\mathrm{nc}} & \text { if } \mathrm{P}<1 \wedge \mathrm{F}>0 \\ 0 & \text { otherwise }\end{cases}
$$

where

$$
\begin{aligned}
& \mathrm{P}=\mathrm{P}\left(\mathbf{r}_{\mathrm{c}}, \mathbf{r}_{\mathrm{n}}\right) \\
& \mathrm{F}=\mathrm{F}\left(\mathbf{r}_{\mathrm{c}}, \mathbf{r}_{\mathrm{n}}\right) .
\end{aligned}
$$

The Equations 2-4 are applied to adhesion to basal membrane where $\mathrm{r}_{n}$ is replaced by $\mathrm{r}_{b m}$, for which the optimal distance is calculated between a cell's center and its nearest basal membrane segment (BMs) as shown in Eq 5. The effective adhesion coefficient is calculated in Eq 6.

$$
\begin{aligned}
& \mathrm{d}_{\mathrm{opt}}\left(\mathbf{r}_{\mathrm{c}}, \mathbf{r}_{\mathrm{bm}}\right)=\left\|\frac{\hat{\mathbf{v}}_{\mathrm{C} \_ \text {bm }}}{\left.\sqrt{\left[\frac{\hat{\mathrm{v}}_{\mathrm{c} \_\mathrm{bm}}^{2}}{a_{\mathrm{c}}^{2}}+\frac{\hat{\mathrm{v}}_{\mathrm{c} \_\mathrm{bm}}}{a_{\mathrm{c}}^{2}}\right]}\right]}\right\|_{2}
\end{aligned}
$$

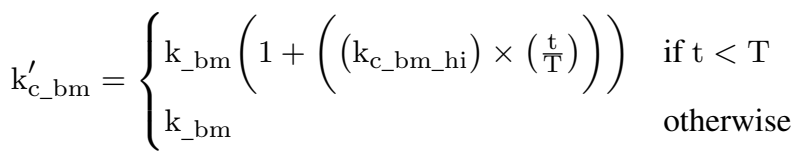

Optimization (Eq 1) is required to maintain a stable connection between adjacent cells. The observation from the existing model shows that the calculation of intercellular pressure (Eq 2) applies to cells that overlap with each other and the cohesion force (Eq 3 ) applies for cells that are in contact with each other. Movement vector $(\mathrm{Eq} 4)$ is calculated for each adjacent cells depending on the intercellular pressure and cohesion force between the cells. The cohesion coefficient $(\mathrm{k})$ regulates the cohesion force, contributing to the estimation of the movement vector. To achieve a new position, the vector is added to the cell's center of mass based on its direction to balance optimal distance.

Adhesion with the basal membrane is estimated to achieve cell migration leading to the wound closure. The calculation of the optimal distance between a cell and a basal membrane is obtained using Eq 5. The Eq 3 and Eq 4 are used to calculate the movement vector and achieve the desired migration. Further parameters used in the above mentioned equations are given in Table 1.

The model developed in this paper reproduces the existing RM model. The algorithm consists of two movement types: A) interaction of basal cells with the basal membrane and B) interaction of a cell with its surrounding cells. The model consists of five circular cells named cell c, n1, n2, n3, and n4. The cells near the basal membrane are considered to be the basal cells performing both the movements.

Basal cells (cell n4, c and n1) first estimate the adhesion to the basal membrane using Eq 1-6. These cells determine a movement vector, which leads to a new position (case A). On the other hand, cell-cell interaction occurs with all the cells involved in the simulation (case B). The cell-cell interaction calculates the vector of the neighboring cells to balance the optimal distance between adjacent cells with their new position (case B).

The optimal balancing needs multiple simulation steps with different coefficient values. The need for different coefficient value is due to varied cell positions. In this algorithm, the 
Tab. 1: Parameter values used in given equations.

\begin{tabular}{llll}
\hline Parameter & Definition & Value & Source \\
\hline$\delta_{p}$ & Overlapping factor & 0.95 & {$[2]$} \\
$\delta_{a d}$ & Adhesion factor & 1.25 & {$[2]$} \\
$\mathrm{k}$ & Cohesion coefficient & $0.2: 0.1: 0.9$ & not in [2] \\
$\mathrm{k}_{-b m}$ & Adhesion coefficient & $0.5: 0.5: 1.5$ & not in [2] \\
$\mathrm{k}_{c \_b m \_} h i$ & High adhesion coefficient & 2.5 & {$[2]$} \\
$\mathrm{T}$ & Threshold for step & 1050 & {$[2]$} \\
\hline
\end{tabular}

simulation is performed with both the movement types, starting with cell $\mathrm{n} 4$, followed by $\mathrm{c}$ and $\mathrm{n} 1$. The simulation continues till the edge cell $\mathrm{n} 1$ reaches the targeted wound closure.

\subsection{Parameter sensitivity analysis}

The model in this paper uses parameter values from Table 1 to calculate (Eq 1-6). The coefficient values for adhesion and cohesion are not provided [2]. So a parameter sensitivity analysis was conducted on these coefficients with known parameters from RM model.

The cohesion coefficient and adhesion coefficient (see Table 1) are applied to cells involved in cell-cell interaction and cell - basal membrane interaction respectively. These interactions achieve cell migration and maintain optimal balance to attain the target wound closure. The outcome reflects the forward cell migration of reepithelialization mechanism which is intact to the basal cells [2].

\section{Results}

\subsection{Reepithelialization model}

The optimal distance balancing process at simulation step $t_{0}$ and $t_{n}$, which implements cell - basal membrane interaction and cell-cell interaction are shown in Table 2 and 3. In this algorithm, the optimal distance is balanced in multiple steps to reach wound closure. Intercellular pressure (Eq 2) between overlapping cells and cohesion force (Eq 3 ) between adjacent cells are calculated. The observation from Table 2 and Table 3 show high pressure and high force when the distance between two cells is less than the optimal distance. Overlapping of cells occur if $\mathrm{P}\left(\mathbf{r}_{\mathrm{c}}, \mathbf{r}_{\mathrm{n}}\right)>1$ and $\mathrm{F}\left(\mathbf{r}_{\mathrm{c}}, \mathbf{r}_{\mathrm{n}}\right)>1$ (see n1_n2 in Table2 and see n4_n3 and c_n2 in Table 3). In Table2, the cohesion force is zero as the condition $\left\|\mathbf{v}_{n c}\right\|_{2}<\delta_{a d h} \times d_{o p t}\left(\mathbf{r}_{c}, \mathbf{r}_{n}\right)$ is not satisfied. The calculated movement vector (Eq 4) pushes the cells away if they overlap and pulls them closer if the cells are not in contact.
The algorithm applies to all the basal cells (with Eq 1 - 6) to calculate their new positions. The simulation results show the two movement types. In the first type, the cells move towards wound closure and, in the second type, the neighbor cells move towards the basal cells. Here, the first type estimates the speed of healing, and the second develops intact tissue. This contributes to the movement of the basal cells towards closure, trying to balance the distance between adjacent cells along the forward path. The simulation results at $t_{0}, t_{1}$ and $t_{n}$ are shown in Fig. 1.

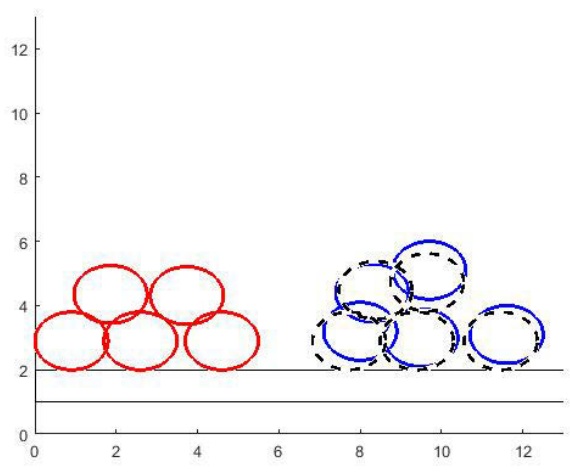

Fig. 1: Simulation results at step $t_{0}, t_{1}$, and $t_{n}$. The initial position of cells at step $t_{0}, t_{1}$ and $t_{n}$ are indicated by blue, black and red colors respectively. The cells are named from right to left as $\mathrm{n} 4, \mathrm{c}$, and $\mathrm{n} 1$ in layer one and as n3 and n2 in layer two.

\subsection{Parameter sensitivity analysis}

To perform parameter sensitivity analysis, a set of adhesion and cohesion coefficients are taken into considerations (see Table 1). In the forward movement, the basal cells interact with each BMs. When the adhesion coefficient is low, the healing rate is slow compared to those with higher coefficients. This comparison is justified with the total number of simulation steps required to reach the target wound closure (see Table 4 ).

When the adhesion coefficient is increased from 0.5 to 1.0, the step size increases. This, in turn, increases cohesion coefficient to balance optimal distance. At $\mathrm{k}_{-} b m=1.5$, the step size is large and the neighboring cells lose contact with basal cells on its way to the targeted closure. Therefore, after analysing the obtained results, $\mathrm{k}_{-} b m=0.5$ and $\mathrm{k}=0.9$ provide better results from the given set of coefficients at $t_{n}$ (see Fig. 1 and Table 2).

The obtained results of the model shows increase in the magnitude of movement vector for higher cohesion coefficient. This explains that increasing the coefficients increases the cellular movement. Thus, the speed of wound healing increases with the cohesion and adhesion coefficients. 
Tab. 2: Results for cell-cell interaction before simulation

\begin{tabular}{lllll}
\hline Cells & n4_n3 & c_n3 & c_n2 & n1_n2 \\
\hline Optimal distance $[\mu m]$ & 1.8 & 1.8 & 1.8 & 1.8 \\
Distance $[\mu m]$ & 2.7586 & 2.1095 & 1.8439 & 1.2369 \\
Intercellular pressure[Pa] & 0.6199 & 0.8106 & 0.9274 & 1.3825 \\
Cohesion force[N] & 0 & 0.8533 & 0.9762 & 1.4553 \\
\hline
\end{tabular}

Tab. 3: Results for cell-cell interaction after simulation, at $t_{n}$

\begin{tabular}{lllll}
\hline Cells & n4_n3 & c_n3 & c_n2 & n1_n2 \\
\hline Optimal distance $[\mu m]$ & 1.8 & 1.8 & 1.8 & 1.8 \\
Distance $[\mu \mathrm{m}]$ & 1.6501 & 1.8176 & 1.6257 & 1.8196 \\
Intercellular pressure[Pa] & 1.0363 & 0.9408 & 1.0519 & 0.9398 \\
Cohesion force[N] & 1.0908 & 0.9903 & 1.1072 & 0.9892 \\
\hline
\end{tabular}

\section{Discussion}

This paper recreates the reepithelialization model which includes two movement types: cell - basal membrane interaction and cell-cell interaction [2]. Here, parameter sensitivity analysis is performed for adhesion and cohesion coefficients. The given equations with known parameters are applied to the algorithm, resulting in balancing the optimal distance with each basal cells and cell migration towards wound closure. The result shows that the speed of cellular migration increases with increasing coefficients.

This work performs parameter sensitivity analysis similar to the previous work [3] but uses a different model to see the effects of sensitivity parameters on healing time. Reepithelialization model performs proliferation, migration, and differentiation, while this work focuses on migration. The observed result shows increasing healing time with an increase in the sensitivity parameter. If the adhesion coefficient is increased, the healing time decreases and in turn increases the cohesion coefficient to balance with the surrounding cells. The presence of proliferation and differentiation provides stable contact between cells using a push-pull mechanism. If multiple cells are present because of proliferation, the required healing time decreases, thereby accepting low sensitivity parameters.

The model with initial settings consider circular cell's with fixed radius, and their positions are assumed to be at the wound edge with the intact surrounding tissue. Since a specific sensitivity parameter is used throughout the simulation,

Tab. 4: Total number of steps required for edge cell $\mathrm{n} 1$ to reach the target closure

\begin{tabular}{llll}
\hline Adhesion coefficient & $\mathbf{0 . 5}$ & $\mathbf{1 . 0}$ & $\mathbf{1 . 5}$ \\
\hline Total steps & 280 & 132 & 76 \\
\hline
\end{tabular}

optimization is not achieved in a single simulation step. This is due to different positioning of the cells.

The absence of movement vector occurs when the basal cells are at an optimal distance. Basal cells start interacting with their nearest basal membrane segment along the targeted closure and migrate in a forward direction, to attain wound closure. This work proposes a future scope for cell proliferation, which can provide better optimization between cells. When the adjacent cells balance optimization, a new cell is introduced between them. The proposal can increase the cell count, thereby building a stable shield of reepithelialization with intact cells.

\section{Conclusion}

This paper implemented a reepithelialization model to study cell migration in the wounded area. To determine the movement vector of the individual cells, a parameter sensitivity analysis was conducted on the parameters with the unknown values. This analysis shows that the speed of movement increases for the basal cells to the closure for higher adhesion coefficient and vice versa for lower coefficient. If the cohesion coefficient is low, the movement of cells towards basal cells is very slow and vice-versa for higher coefficients. Thus showing the influence of sensitive parameters in cell migration.

\section{Author Statement}

Research funding: This study was partially supported by the German Federal Ministry of Research and Education (BMBF under project OWID/IP1 CoHMED grant number FKZ 13FH5I01IA) with ATMOS MedizinTechnik. Conflict of interest: Authors state no conflict of interest.

\section{References}

[1] Raja, S.K., Garcia, M.S. and Isseroff, R.R., 2007. Wound reepithelialization: modulating keratinocyte migration in wound healing. Front Biosci, 12(3), pp.2849-2868.

[2] Safferling, K., Sütterlin, T., Westphal, K., Ernst, C., Breuhahn, K., James, M., Jäger, D., Halama, N. and Grabe, N., 2013. Wound healing revised: a novel reepithelialization mechanism revealed by in vitro and in silico models. J Cell Biol, 203(4), pp.691-709.

[3] McQueen, A., Parente, J.D., McGinty, S. and Moeller, K., 2019. Parameter Search to Find Ranges of Activation and Inhibition of Wound Healing Rate in a Mathematical Model with Introduced Photobiomodulation. In World Congress on Medical Physics and Biomedical Engineering 2018 (pp. 819822). Springer, Singapore. 